\title{
Initial Investigation of the Effects of an Experimentally Learned Schema on Spatial Associative Memory in Humans
}

\author{
${ }^{\circledR}$ Mariët van Buuren, ${ }^{1,2}$ Marijn C.W. Kroes, ${ }^{3}$ Isabella C. Wagner, ${ }^{1,2}$ Lisa Genzel, ${ }^{4}{ }^{-}$Richard G.M. Morris, ${ }^{4}$ \\ and Guillén Fernández ${ }^{1,2}$ \\ ${ }^{1}$ Donders Institute for Brain, Cognition and Behaviour, Centre for Cognitive Neuroimaging, and ${ }^{2}$ Departement for Cognitive Neuroscience, Radboud \\ University Nijmegen Medical Centre, 6500 HB, Nijmegen, The Netherlands, ${ }^{3}$ Donders Institute for Brain, Cognition and Behaviour, Radboud University \\ Nijmegen, 6500 HB, Nijmegen, the Netherlands, and ${ }^{4}$ Centre for Cognitive and Neural Systems, The University of Edinburgh, EH8 9JZ, Edinburgh, United \\ Kingdom
}

Networks of interconnected neocortical representations of prior knowledge, "schemas," facilitate memory for congruent information. This facilitation is thought to be mediated by augmented encoding and accelerated consolidation. However, it is less clear how schema affects retrieval. Rodent and human studies to date suggest that schema-related memories are differently retrieved. However, these studies differ substantially as most human studies implement pre-experimental world-knowledge as schemas and tested item or nonspatial associative memory, whereas animal studies have used intraexperimental schemas based on item-location associations within a complex spatial layout that, in humans, could engage more strategic retrieval processes. Here, we developed a paradigm conceptually linked to rodent studies to examine the effects of an experimentally learned spatial associative schema on learning and retrieval of new object-location associations and to investigate the neural mechanisms underlying schema-related retrieval. Extending previous findings, we show that retrieval of schema-defining associations is related to activity along anterior and posterior midline structures and angular gyrus. The existence of such spatial associative schema resulted in more accurate learning and retrieval of new, related associations, and increased time allocated to retrieve these associations. This retrieval was associated with right dorsolateral prefrontal and lateral parietal activity, as well as interactions between the right dorsolateral prefrontal cortex and medial and lateral parietal regions, and between the medial prefrontal cortex and posterior midline regions, supporting the hypothesis that retrieval of new, schema-related object-location associations in humans also involves augmented monitoring and systematic search processes.

Key words: fMRI; medial prefrontal cortex; memory retrieval; schema; spatial associative memory

\section{Introduction}

The presence of prior knowledge, schema, promotes memory for new, congruent information (Bartlett, 1932; Anderson, 1981). Recent neurobiological hypotheses have further defined a schema as a pre-existing network of interconnected neocortical representations (Wang and Morris, 2010; Kroes and Fernández, 2012; van Kesteren et al., 2012), and data ranging from a biomolecular level in rodents to patient work and education in humans (Tse et al., 2007, 2011; Kumaran et al., 2009; van Kesteren et al., 2010a, 2013, 2014; Wang et al., 2012; McKenzie et al., 2013; Warren et al., 2014) suggest that processes underlying memory encoding and consolidation are facilitated by schema. These studies

\footnotetext{
Received June 11, 2014; revised 0ct. 24, 2014; accepted 0ct. 29, 2014.

Author contributions: M.v.B., M.C.W.K., L.G., R.G.M.M., and G.F. designed research; M.v.B. and I.C.W. performed research; M.v.B. analyzed data; M.v.B., M.C.W.K., R.G.M.M., and G.F. wrote the paper.

This project was supported by a grant by the European Research Council (ERC R0001075) to R.G.M.M. and G.F. The authors declare no competing financial interests.

Correspondence should be addressed to Dr Mariët van Buuren, Donders Institute for Brain Cognition and Behaviour, Centre for Cognitive Neuroimaging, P0 Box 9101, NL-6500 HB Nijmegen, The Netherlands. E-mail: m.vanbuuren@donders.ru.nl.

M.C.W. Kroes's present address: Center for Neuroscience, Department of Psychology and Center for Neural Science, New York University, New York, NY 10003.

DOI:10.1523/JNEUROSCI.2365-14.2014

Copyright $\odot 2014$ the authors $\quad 0270-6474 / 14 / 3416662-09 \$ 15.00 / 0$
}

indicate that this facilitation is mediated by the medial prefrontal cortex (mPFC), and its interactions with the medial temporal lobe (MTL) and posterior representational areas.

The effects of schema on memory retrieval are less clear. In rodents, a spatial associative schema of flavor-location associations in an event arena enabled rapid assimilation of new associations, resulting in hippocampally independent retrieval, and a dependency on mPFC activity for the retrieval of old schema associations and new associations (Tse et al., 2007, 2011). In humans, enhanced mPFC activity and strengthened interactions between the $\mathrm{mPFC}$ and posterior representational areas during retrieval of schema congruent information has been reported (van Kesteren et al., 2010b), suggesting that schema-related memories are differently retrieved. The role of the hippocampus in schema effects on memory retrieval in humans is less clear as the hippocampus may continue to contribute to retrieval if intact or changes may not be detectable by imaging methods in humans. Critically, to date, schema studies in humans and rodents differ substantially. Although most human studies implement pre-experimental academic or world-knowledge as schemas, and tested item and nonspatial associative memory, animal studies have used intraexperimentally learned schemas based on itemlocation associations within a complex spatial layout. To better 

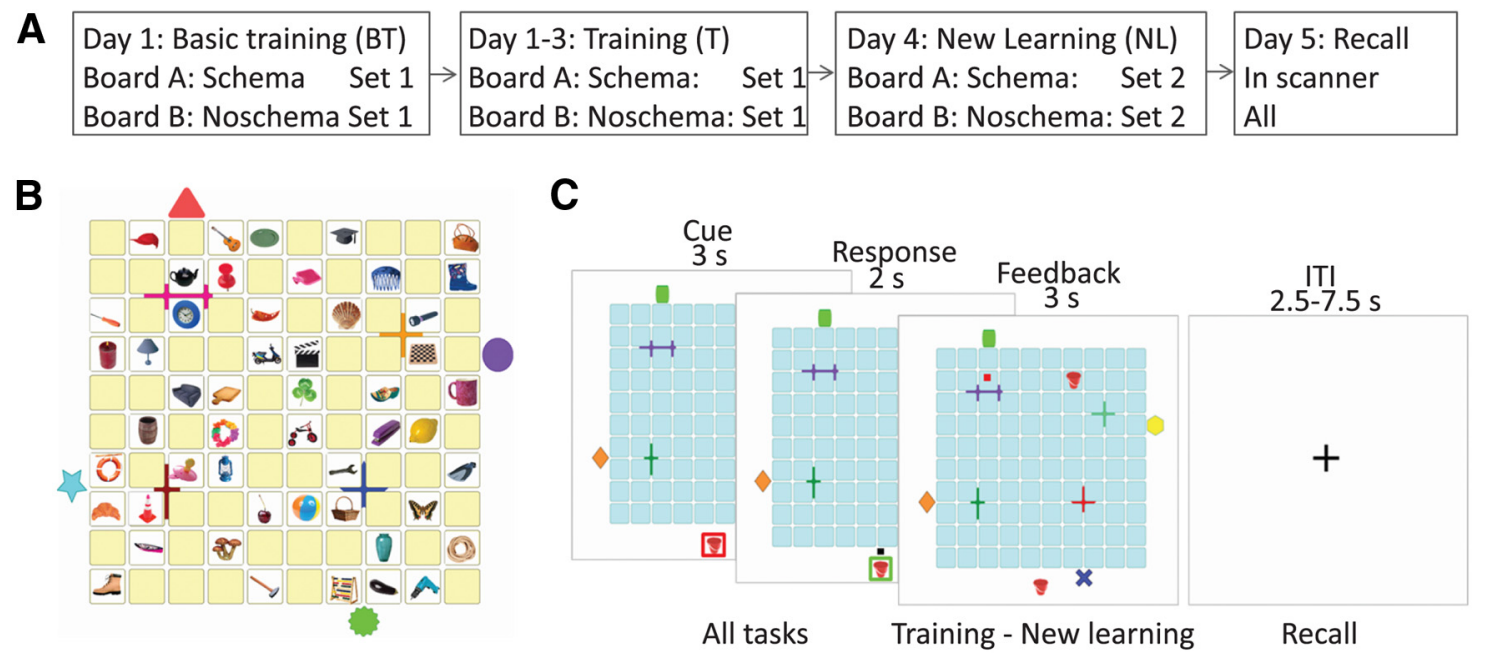

Figure 1. Experimental design. $A$, Experimental procedure. On day 1, subjects performed a basic training in which they had to learn associations between 50 objects and locations (Set 1 ) on each of the boards. On the same day, a more advanced training was performed in which subjects again learned associations between the same 50 objects and locations. This training was repeated on day 2 and 3. On day 4, 50 new object-location associations (Set 2) were introduced per board and $24 \mathrm{~h} \mathrm{later} \mathrm{recall} \mathrm{for} \mathrm{all} \mathrm{associations} \mathrm{was} \mathrm{tested} \mathrm{in} \mathrm{the} \mathrm{MR} \mathrm{scanner.} \mathrm{The} \mathrm{associations} \mathrm{of} \mathrm{Set} 1$ on the schema board remained consistent between sessions, whereas the association of Set 1 on the noschema board changed before the training and new learning sessions. $\boldsymbol{B}$, Example of a board with one set of associations displayed and colored graphical forms outside and inside the board as landmarks. C, Timing of a trial of the (basic) training, new learning session, and recall session. The cue and response period were similar in all sessions. In the training and new learning sessions, feedback was presented on the screen immediately after response for the remaining trial duration (left), whereas during the recall session, the cursor turned gray after response for the remaining response period, followed by an intertrial interval (ITI; right).

align these lines of research and to further investigate how schema affects retrieval in humans, it appears crucial to design a controlled study in which the intraexperimental schema is based on item-location associations within a spatial layout. Particularly in humans, such a complex spatial task may hold greater mnemonic demands and evoke elaborative and strategic retrieval processes (Anderson, 1984; Ghosh and Gilboa, 2014) that could contribute to schema effects on memory retrieval. These processes have been associated with parietal and lateral prefrontal areas that could support retrieval indirectly (Simons and Spiers, 2003; Ciaramelli et al., 2008) or by retrieval processes specifically linked to schema representations. To investigate such potential processes, we extended previous studies (Tse et al., 2007, 2011; van Kesteren et al., 2010b) by using whole-brain approaches instead of focusing mainly on the midline structures and MTL.

Here, we investigate the impact of schema on learning and retrieval of new object-location associations, as well as the neural correlates underlying such retrieval. Subjects were instructed to memorize associations between objects and locations within two spatial layouts: a schema and a noschema layout. On 3 consecutive days, subjects were trained on associations in both conditions. In the schema condition, associations were formed and stabilized within a spatial layout that remained consistent over sessions. In the noschema condition, however, the objectlocation associations changed across sessions. On day 4, each of the two spatial layouts was extended with new associations and $24 \mathrm{~h}$ later, on day 5 , recall for all associations were tested while measuring brain activity with fMRI.

We expect better learning and memory for object-location associations that can be successfully related to the learned schema. Moreover, we expect increased mPFC activity and connectivity with posterior representational areas during retrieval of schema-related associations. Additionally, as the schema may facilitate strategic search processes supporting retrieval, we presume augmented processing within parietal and lateral prefrontal areas.

\section{Materials and Methods}

\section{Participants}

Complete datasets were acquired of 33 right-handed healthy subjects. The subjects were recruited from the university campus in Nijmegen and received monetary compensation or course credits for their participation. In addition, they could earn extra money based on performance. None of the subjects received psychotropic medication or had any contraindication for MRI, or a self-reported current or past neurological, psychiatric, or substance use disorder. One subject was excluded due to excessive head motion during the fMRI session $(>4 \mathrm{~mm})$, and 10 were excluded because they did not have sufficient numbers of trials $(\geq 9)$ in conditions used for fMRI analyses, as some subjects had too good and others too poor memory performance. This left 22 subjects (ages 19-32 years, mean 21.9 years; 13 male) for analyses with 27.1 trials per condition on average. All gave written informed consent. The study was performed in accordance with the Declaration of Helsinki and was approved by the institutional Medical Research Ethics Committee (CMO Region, Arnhem-Nijmegen).

\section{Task and procedure}

The overall task of the subjects was to memorize associations between pictures of everyday objects and their locations within two $10 \times 10$ "card" boards presented on a computer screen: a schema board and a noschema board. Subjects came to our laboratory on 5 consecutive days (Fig. 1A). On the first day, they were introduced to the task in a basic training session. In this session, they learned the locations of 50 objects per board (Set 1). In the afternoon, they returned for a more advanced training session, which was repeated on the second and third day. During these advanced training sessions, they again needed to learn the associations between the same 50 objects and their locations on each board. Critically, on the schema board, the associations of Set 1 remained consistent over training sessions, enabling the buildup of a spatial associative schema. In contrast, on the noschema board, the objects and their possible locations within Set 1 remained fixed, whereas the associations between individual objects and their specific locations changed on every training session. On day 4 , the 50 remaining object-location associations (Set 2) were introduced for each board in addition to Set 1 associations during a final learning session (new learning session). The associations of Set 1 on the noschema board again changed. On day 5 , subjects were 
asked to retrieve the correct location of all 100 objects on each board while their brain activity was measured using fMRI.

Basic training. During an initial basic training session, subjects were instructed to learn 50 object-location associations initially on one of the boards. At the start of the task, one of the cardboards with its 50 objects was displayed on the screen for $1.5 \mathrm{~min}$ to increase learning speed. The 50 remaining cards were displayed face down (Fig. 1B). After viewing the board, the first trial started with the presentation of a cue, one of the objects, in a red frame presented below the board, the board presented with all cards facing down. After $2 \mathrm{~s}$, the frame turned green and a black square cursor appeared randomly at one of the four sides of the board. By starting randomly at one of the four sides, subjects could not use pure egocentric stimulus-response trajectory learning. In this response period of $2 \mathrm{~s}$, subjects had to select the card (i.e., location) they believed hold the same object by moving to that card and pressing the left button on a trackball (Kensington, Orbit Optical Trackball). After selecting a card, feedback was presented on the screen for $3 \mathrm{~s}$ plus the remaining response period (Fig. 1C). If the incorrect card was selected, the cursor turned red and the object was displayed at the correct location. If the correct card was selected, the object was shown at that location. After every 50 trials, a rest block of $30 \mathrm{~s}$ was inserted during which a fixation cross was presented on screen. The task consisted of three learning cycles, in which every association was presented once per cycle. After three learning cycles were completed, the subjects had to learn the other board and set of 50 associations. Order of schema and noschema board was counterbalanced across subjects.

Training. On 3 consecutive days, subjects returned for training sessions, each lasting $\sim 45 \mathrm{~min}$. Each session was identical and similar to the basic training session. However, the boards (conditions) were presented in a more rapidly alternating fashion. That is, at the start of the task, both boards with their objects were presented after each other for $1.5 \mathrm{~min}$, and during the task, the boards alternated every five trials. Such interleaved learning is thought to reduce interference (McClelland et al., 1995). The start condition was counterbalanced over subjects and sessions. On the next day and the third day, the training session was repeated. The order of presentation and assignment of the objects to the possible locations on the noschema board were randomized for each subject and session. After the last training on day 3 , a recall task (see description recall task in Recall in scanner, below) was performed to familiarize subjects with the actual cued-recall setup. During this test, memory for all trained associations (Set 1) was tested.

New learning. On day 4, subjects returned to our laboratory once again to learn the 50 remaining object-location associations per board (Set 2) in addition to Set 1 associations. The 50 new objects were placed at the remaining 50 locations not used for Set 1 . Associations of Set 1 on the noschema board changed again. At the beginning of this session, both boards with all objects were presented after each other on the screen, each for $2.5 \mathrm{~min}$. During the task, the boards alternated every five trials and the start condition was counterbalanced over subjects. Similar to the training sessions, after the initial presentation of the boards, the first trial started with presentation of a cue below the board $(3 \mathrm{~s})$, followed by a response period ( $2 \mathrm{~s}$ ) and feedback ( $3 \mathrm{~s}$; Fig. 1C). Each object was presented once during each of three learning cycles. In total, the session lasted $100 \mathrm{~min}$.

Recall in scanner. Twenty-four hours $(\mathrm{SD}=1 \mathrm{~h})$ later, memory for all 100 object-location associations on each board was tested in the MR scanner. Before the start of the task, the instructions were repeated and subjects were asked how many hours they had slept the previous night (mean $\pm \mathrm{SD}, 7.8 \pm 1.4 \mathrm{~h}$ ). Then, subjects were positioned in the MR scanner, where they lay supine with a trackball on their abdomen (Kensington, Orbit Optical Trackball, in house adapted for MRI compatibility) to perform the task. They could view the task on the screen through a mirror mounted on the head coil. In this test, each object was presented once and sequentially as a cue below the board in a red frame for $3 \mathrm{~s}$. The board was displayed with the cards facing down. For each cue, subjects were instructed to select the correct card (location) within $2 \mathrm{~s}$ after cue offset, similar to the training and new learning sessions. However, after selection of the card, no feedback was presented. Instead, the cursor turned gray for the remaining trial duration, followed by a $2.5-7.5 \mathrm{~s}$ intertrial interval (fixation cross, mean $5 \mathrm{~s}$; Fig. 1C). Again, the boards alternated every five trials during the task and the start condition was counterbalanced over subjects. Resting blocks were inserted after every 25 trials during which a fixation cross was presented for $30 \mathrm{~s}$. The task duration was $\sim 40 \mathrm{~min}$.

Item recognition task. After scanning, an item recognition memory task was presented to the subjects outside the scanner to test whether the subjects' ability to recognize the objects differed between the schema and noschema board. In this task, 25 objects randomly selected from each board and set were intermixed with 50 comparable but entirely novel objects, and sequentially presented on screen for $3.5 \mathrm{~s}$. Subjects had to indicate by appropriate button press (within the presentation time) whether they remembered having seen the object during the experiment with high confidence ("sure old") or with low confidence ("unsure old"), or whether they thought that they had not seen the object during the experiment with low confidence ("unsure new") or with high confidence ("sure new").

\section{Stimuli, stimulus presentation, board design}

Stimuli. In total, 250 colored pictures of simple, everyday objects were selected from the Hemera Photo-Objects database (Hemera Technologies). All objects were easy to name and distinctive. These pictures of objects (called objects in the remaining text) were divided into five sets of 50 each, all sets comparable for color and content (transportation objects, food items, household items, sport and play items, etc). These sets were assigned to each subject and condition (Set 1 schema, Set 1 noschema, Set 2 schema, Set 2 noschema, novel in item recognition task) in a counterbalanced manner.

Board design. The $10 \times 10$ "boards" were created in Adobe Illustrator CS5.0 and differed only in landmarks (see below) and color of the cards' back; one condition had cards with yellow backs and the other blue. Around and within the boards, colored graphical forms, "landmarks" were located. These landmarks could be used as reference points when memorizing the locations of objects on the boards similar to the salient objects in and near the event arena (Tse et al., 2007). The colors of the landmarks as well as the shape of the outer landmarks differed between boards, but the placement was the same. Assignment of the boards to the schema and noschema condition was counterbalanced over subjects.

Stimuli presentation. Next, the objects were assigned to the locations on the boards as follows. Two sets of coordinates of locations were developed, each consisting of two parts, one part of coordinates for the objects of Set 1 and one for Set 2. The locations were selected in such a way that the two sets were divided equally over the quadrants of the boards. The assignment of the sets of coordinates and its respective parts to the specific board (schema and noschema) and set (Set 1 and Set 2) was counterbalanced over subjects. Next, for each subject, each object of a specific set and condition was randomly assigned to one of the locations of that specific set and condition before start of the basic training session (Set 1) and new learning session (Set 2). In case of the objects of Set 1 of the noschema board, this randomization was repeated before every training session and the new learning session. In addition, the order of presentation of the objects of both the schema and noschema board was randomized for each session and each learning cycle. All versions of the task were programmed in and presented using Presentation 16.1 (NeuroBehavioral Systems).

\section{Behavioral analyses}

The behavioral data consisted of scores for the accuracy of remembering the correct locations of objects, and these were analyzed using IBM SPSS Statistics 20. Repeated-measures ANOVAs were conducted to test the effects of schema (schema, noschema), session (training 1,23) and cycle $(1,2,3)$ on learning performance (correct responses) during the training sessions. Next, we tested the impact of having a reliable schema on the learning of the new, additional associations (Set 2) on day 4 using a repeated-measures ANOVA with schema (schema, noschema) and cycle $(1,2,3)$ as within-subject factors. To test whether possessing a schema affected memory for the newly learned associations during the recall task, a paired-sample $t$ test was performed. In addition, we performed a repeated-measures ANOVA to test the effects of schema (schema, noschema) and memory (correct, incorrect) on reaction times (RTs) for 
the newly learned associations during the recall task. In addition, we conducted paired-sample $t$ tests comparing the RT for the correctly recalled schema-defining associations (Set 1) with the RT for the correctly recalled noschema associations of Set 1, as well as with the schema associations of Set 2. Furthermore, data from the item recognition task was investigated with a repeated-measures ANOVA with schema (schema, noschema) and set (Set 1, Set 2) as within-subject factors to test for differences in object recognition between conditions and sets.

\section{MRI data acquisition}

Participants were scanned using a Siemens Magnetom Skyra 3 tesla MR scanner equipped with a 32-channel phased array head coil. A total of 1098 blood oxygen level-dependent scans were acquired using a T2*weighted gradient-echo, multiecho echoplanar imaging (EPI) sequence (Poser et al., 2006) with the following parameters: repetition time (TR) $=$ $2100 \mathrm{~ms}$; echo time $(\mathrm{TE}) 1=8.5 \mathrm{~ms}$, TE $2=19.3 \mathrm{~ms}$, TE $3=30 \mathrm{~ms}$, TE $4=$ $41 \mathrm{~ms}$; flip angle $=90^{\circ}$; matrix size $=64 \times 64$; field-of-view $(\mathrm{FOV})=224$ $\times 224 \times 119$; slice thickness $=3 \mathrm{~mm}$; slice gap $=0.51 \mathrm{~mm}$; 34 slices, acquired in ascending order. To allow T1 saturation to reach equilibrium, the first six scans were discarded.

After acquisition of the functional data, a T1-weighted structural image of the whole brain was made using a magnetization prepared, rapidacquisition gradient echo sequence (parameters: $\mathrm{TR}=2300 \mathrm{~ms}$; $\mathrm{TE}=$ $3.03 \mathrm{~ms}$; flip angle $=8^{\circ}$; matrix size $=256 \times 256$; FOV $=256 \times 256 \times$ 256; slice thickness $=1 \mathrm{~mm}$; 192 sagittal slices).

\section{MRI data preprocessing}

Raw multiecho data were first combined using in-house build MATLAB 7.9 software (MathWorks). This software used 32 scans acquired during resting-state before start of the task to determine the optimal weighting of echo-times for each voxel (after applying a smoothing kernel of $3 \mathrm{~mm}$ full-width at half-maximum), by calculating the contrast-to-noise ratio for each echo per scan. Next, motion correction was performed on the first echo by estimating iterative rigid body realignment to minimize the residual sum of squares between the first echo of the first scan and all other scans. These estimated parameters were applied to all other echos, thereby realigning all echos to the first echo of the first scan. Then, the calculated optimal echo time weightings were used to combine the four echo images of each scan into single images. These combined images were further preprocessed and analyzed using SPM8 (http://www.fil.ion. ucl.ac.uk/spm).

The individual structural image was coregistered to the mean functional scan using mutual information optimization. Next, using unified segmentation, the structural scan was segmented and the normalization parameters were estimated. Subsequently, using these parameters, all scans were transformed into Montreal Neurological Institute (MNI) space, as defined by the SPM8 MNI T1 template (resampled voxel size of functional data $=3.5 \times 3.5 \times 3.5 \mathrm{~mm}$ ). Finally, the functional scans were smoothed using a 3D Gaussian filter $(8 \mathrm{~mm}$ full-width at half-maximum).

\section{fMRI data analyses}

The preprocessed functional images were analyzed using a general linear model regression analysis in which the following events were included as regressors of interest: correct trials of Set 1 of the schema condition, correct trials of Set 1 of noschema condition, correct trials of Set 2 of schema condition, correct trials of Set 2 of noschema condition, incorrect trials of Set 1 of noschema condition, incorrect trials of Set 2 of schema condition, and incorrect trials of Set 2 of noschema condition. For these regressors, a parametric modulation of reaction time was included to control for variation in response time. No separate regressor of interest was included for incorrect trials of Set 1 of the schema, as performance on these trials was, as intended, very good, resulting in low numbers of incorrect trials for all subjects. Instead, a regressor was included modeling the incorrect trials of Set 1 of the schema condition together with the misses of all conditions. All regressors were time-locked to the onset of the cue and modeled by convolving a box-car function of $5 \mathrm{~s}$ (cue presentation + maximum response period) with a canonical hemodynamic response function (Friston et al., 1995). To correct for head motion, the six realignment parameters were included in the design matrix as regres- sors of no interest. A high-pass filter was applied to the data (cutoff $150 \mathrm{~s}$ ) to remove low-frequency fluctuations in the signal. Subsequently, for each subject contrast images were created for each condition of interest compared with baseline. These contrast images were submitted to random effects second-level analyses.

As second-level analyses, two repeated-measures, flexible factorial ANOVAs were conducted with "subject" modeled as a factor. First, we performed a two-way ANOVA with the within-subject factors schema (schema, noschema) and set (Set 1, Set 2) to examine activity during correct retrieval of all associations on both boards. Second, a two-way ANOVA with schema (schema, noschema) and memory (correct, incorrect) as within-subject factors was conducted to investigate brain activity related to retrieval of the newly learned associations. Note that this analysis only includes the first-level contrast images of retrieval of Set 2 associations.

Next, we performed psychophysiological interaction analyses (PPI; Friston et al., 1997) using SPM8 to assess changes in connectivity associated with memory for the newly learned schema-related associations (Set 2). A PPI shows changes in connectivity of a seed region (physiological factor) with the rest of the brain modulated by a psychological factor (task conditions). Here, we defined two seed regions. The first seed region was defined as a sphere with an $8 \mathrm{~mm}$ radius and centered at the peak of activation within the frontal cortex, which showed increased activity in relation to retrieval of newly learned schema associations (right middle frontal gyrus: $x, y, z=24,18,52$; see Results for more detail). The second seed region was defined as a sphere with an $8 \mathrm{~mm}$ radius and centered in the mPFC, coordinates $x, y, z=-6,34,12$, based on a previous study of schema-related retrieval (van Kesteren et al., 2010b). For each subject and each seed region, the time course within the seed region was extracted and adjusted for average activation during the task (i.e., F-contrast showing effects of task). Separately for the schema and noschema condition, we conducted a PPI analysis computing which areas showed increased connectivity (i.e., positive interaction) with the seed region (i.e., physiological factor) during correct retrieval compared with incorrect retrieval (i.e., psychological factor). Subsequently, these resulting individual contrast images of the two PPI analyses of each seed region were submitted to second-level one-sample $t$ tests.

Unless otherwise specified, all second-level analyses were tested for significance using cluster-inference with a cluster-defining threshold of $p<0.001$ and a cluster-probability of $p<0.05$ familywise error (FWE) corrected for multiple comparisons.

\section{Results}

\section{Behavior}

Schema-defining associations (Set 1) were well learned by the end of day 3 (mean $94.9 \%$ correct), indicating the development of a spatial associative schema (Fig. 2). As expected, the schemadefining associations were better learned than the varying associations on the noschema board (main effect schema; $F_{(1,21)}=$ 985.8, $p<0.0005)$ and this difference increased over sessions (interaction schema $\times$ session; $F_{(2,42)}=24.8, p<0.0005$ ). However, Set 1 associations on the noschema board were also learned within each session (main effect cycle; $F_{(1.6,32.8)}=233.0, p<$ $0.0005)$ and this became faster over sessions (interaction session $\times$ cycle; $\left.F_{(2.6,55.1)}=5.2, p=0.004\right)$, indicating a training effect for the task demands at hand (Fig. 2). The effect of possessing a schema on learning extended beyond this more general training effect, as the associations on the schema board were better learned than on the noschema board (see the above mentioned main effect of schema and interaction schemaxsession).

As predicted, subjects learned new associations (Set 2) on day 4 on the schema board better than on the noschema board (main effect schema; $F_{(1,21)}=25.2, p<0.0005$; no interaction schema $\times$ cycle, $p=0.397$; Fig. 2). Moreover, when memory was tested $24 \mathrm{~h}$ later on day 5 , there was better memory for the newly learned associations on the schema board relative to the noschema board $\left(t_{(21)}=4.6, p<0.0005 ;\right.$ Fig. 2$)$. However, there was a trade-off for 
this accuracy as subjects responded slower to the newly learned associations on the schema board than on the noschema board for both correct (mean RT $\pm \mathrm{SD}$ : schema $=1360.0 \pm 97.1 \mathrm{~ms}$, noschema $=$ $1349.4 \pm 82.1 \mathrm{~ms}$ ) and incorrect trials $($ mean RT \pm SD: schema $=1482.2 \pm 94.5$ $\mathrm{ms}$, noschema $=1429.0 \pm 112.8 \mathrm{~ms}$; main effect schema; $\left.F_{(1,21)}=11.0, p=0.003\right)$. Of note, they were slower on incorrect than on correct trials (main effect memory; $\left.F_{(1,21)}=45.5, p<0.0005\right)$, but no interaction between these factors was observed ( $p=0.139$ ). Furthermore, pairedsample $t$ tests revealed faster response times for correct retrieval of the schemadefining associations (mean $\mathrm{RT} \pm \mathrm{SD}=$ $1328.4 \pm 86.3 \mathrm{~ms}$ ) compared with correct retrieval of both noschema associations of Set 1 (mean RT $\pm \mathrm{SD}=1363.8 \pm 80.7 \mathrm{~ms}$; $\left.t_{(21)}=2.33, p=0.030\right)$ and the newly learned schema-related associations (Set 2 ; mean $\mathrm{RT} \pm \mathrm{SD}$, see above; $t_{(21)}=2.77$, $p=0.012$ ).

Turning to recognition memory for the objects used in the task, $>90 \%$ of the ratings on the item recognition memory task were "high confident," as well as being mainly correct for both the schema and the noschema condition, and no differences were observed between conditions for either set (no main effect schema; $p=$ 0.631 ; or schema $\times$ set interaction; $p=0.875$; mean percentage correct \pm SD: Set 1 , schema $=97.8 \pm 4.0 \%$, noschema $=97.1 \pm$ $7.1 \%$; Set 2 , schema $=91.5 \pm 14.1 \%$, noschema $=91.1 \pm 14.3 \%$ ). Not surprising, recognition memory performance was better for Set 1 than Set 2 (main effect set; $F_{(1,21)}=8.4, p=0.009$ ).

To examine whether the results were influenced by the exclusion of subjects whose performance did not yield enough trials for the fMRI analyses (see Materials and Methods), all analyses were repeated with inclusion of these subjects $(N=32)$. All reported main and interaction effects remained significant in these analyses, and no new significant results were observed.

Together, these findings suggest that the existence of a schema facilitates learning and retrieval of new, related associations, but not item recognition memory. Furthermore, processing of these associations appears more prolonged, whereas processing of the schema-defining associations is faster.

\section{fMRI: activation}

To assess activity during correct retrieval of all associations on both boards, we performed a two-way ANOVA with the factors schema (schema, noschema) and set (Set 1, Set 2). Note that memory (correct, incorrect) was not included as a factor in this analysis, as the incorrect trials of Set 1 on the schema board could not be modeled separately due to low trial numbers caused by high performance, as intended (but see below the next paragraph for further analysis separating correct and incorrect trials). Retrieval of associations on the schema board as compared with the noschema board was associated with more activity within the mPFC, and anterior and middle cingulate cortex (Table 1). However, this main effect of schema was partly qualified by an interaction due to a larger schema effect in Set 1 relative to Set 2 (interaction schema $\times$ set) within the $\mathrm{MPFC}$ and anterior cingulate cortex, as well as within the posterior cingulate cortex and angular gyrus (Table 1). This pattern of results suggests that ac- -schema set1

Accuracy - noschema set1 - schema set2 $\rightarrow$ noschema set2

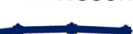

\begin{tabular}{|c|c|c|c|c|c|}
\hline \multirow[b]{2}{*}{ Brain region } & \multicolumn{3}{|c|}{ MNI coordinates } & \multirow[b]{2}{*}{ Z-score } & \multirow[b]{2}{*}{ Voxels } \\
\hline & $x$ & $y$ & $z$ & & \\
\hline \multicolumn{6}{|l|}{ Schema:schema > noschema } \\
\hline L anterior cingulate cortex & 0 & 14 & -10 & 5.40 & 585 \\
\hline R anterior cingulate cortex & 4 & 38 & -4 & 4.96 & \\
\hline R superior frontal gyrus & 4 & 56 & 4 & 4.76 & \\
\hline L cingulate cortex & 0 & -14 & 42 & 4.72 & 189 \\
\hline \multicolumn{6}{|l|}{ Interaction schema $\times$ set } \\
\hline R anterior cingulate cortex & 4 & 38 & -4 & 6.03 & 1265 \\
\hline R superior frontal gyrus & 4 & 63 & 14 & 6.00 & \\
\hline R superior frontal gyrus & 4 & 52 & 4 & 5.91 & \\
\hline $\mathrm{R}$ middle temporal gyrus & 66 & -46 & 4 & 5.07 & 164 \\
\hline R angular gyrus & 56 & -60 & 24 & 4.83 & \\
\hline L posterior cingulate cortex & 0 & -21 & 42 & 4.74 & 107 \\
\hline L precuneus & -4 & -52 & -32 & 4.64 & 74 \\
\hline L angular gyrus & -56 & -66 & 28 & 4.62 & 273 \\
\hline L supramarginal gyrus & -63 & -46 & 21 & 4.47 & \\
\hline L angular gyrus & -56 & -52 & 21 & 4.32 & \\
\hline Cerebellum & -24 & -80 & -35 & 4.43 & 67 \\
\hline
\end{tabular}

Clusters showing significant activity during correct retrieval of all associations (Set 1, Set 2). MNI coordinates represent the location of the peak voxels. The peak voxels of each cluster with the cluster size are followed by separate ( $>8 \mathrm{~mm}$ apart) maxima (maximum of 3 ) within the cluster. First part lists areas showing increased activity during correct retrieval of associations on the schema compared to the noschema board. Second part shows regions with increased activity during incorrect as compared to correct retrieval in the schema relative to the noschema condition. Cluster-defining threshold of $p<0.001$ and $p<0.05$ FWE-corrected critical cluster size of 62 voxels. L, Left; R, right.

tivity within the mPFC, together with activity of the anterior and posterior cingulate cortex and angular gyrus, was related to retrieval of the schema-defining associations (Set 1) rather than retrieval of the newly learned associations (Set 2).

We also conducted a second two-way ANOVA for newly learned associations only, with the factors schema (schema, noschema) and memory (correct, incorrect). Comparing correct retrieval to incorrect retrieval (main effect of memory) revealed increased activity within the anterior and posterior midline regions, angular gyrus bilaterally, putamen and middle temporal areas $(p<0.05$ FWE-corrected, voxelwise interference; Fig. 3A; Table 2). Hippocampal activity was also observed when applying small volume correction (SVC; $p<0.05$, FWE-corrected at cluster level) using a mask of the right and left hippocampus based on the Anatomical Automatic Labeling Atlas (Tzourio-Mazoyer et 
A
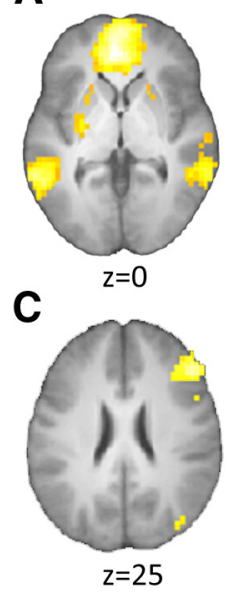
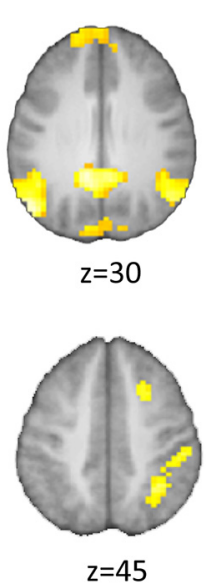

B

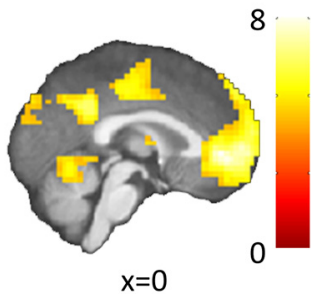

8

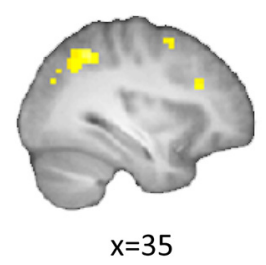

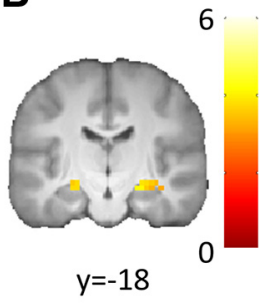

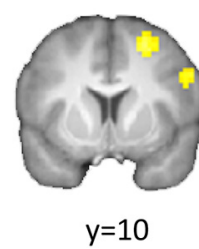

5

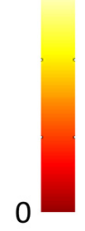

Figure 3. Brain activity during retrieval of newly learned associations (Set 2). A, Main effect of memory. Areas showing increased activity during correct retrieval as compared with incorrect retrieval of associations on the schema and noschema board; $p<0.05$ FWE-corrected for whole brain. $\boldsymbol{B}$, Main effect of memory within hippocampus. Hippocampus showed increased activity during correct retrieval as compared with incorrect retrieval on the schema and noschema board. SVC, $p<0.05$-FWE corrected at cluster level, using a mask of the right and left hippocampus based on the Anatomical Automatic Labeling Atlas. C, Main effect of schema. Areas showing increased activity during retrieval (correct, incorrect) of schemarelated associations as compared with associations on noschema board. Cluster-defining threshold of $p<0.001$ and $p<$ 0.05 FWE-corrected critical cluster size of 62 voxels. Activations overlaid on a mean brain-extracted anatomical image; left $=$ left. Color bar represents $t$ values.

al., 2002; peak coordinates of local maxima, $Z$-value and cluster size: $x, y, z=-14,-4,-14 ; Z=5.72 ; 45$ voxels: $x, y, z=-10$, $-35,4 ; Z=4.99 ; 13$ voxels: $x, y, z=18,-4,-15 ; Z=4.77 ; 76$ voxels; Fig. $3 B$ ). Next, we tested for the main effect of schema by comparing schema board associations with the noschema board associations. This comparison yielded greater activity within the right middle frontal, inferior frontal, and superior parietal gyri (Fig. 3C; Table 2). In addition, we revealed more activity during incorrect as compared with correct retrieval in the schema relative to the noschema condition in the right inferior and superior frontal gyrus ("negative interaction Schema by Memory"; Table 2). However, we did not find evidence for an interaction of schema with memory in which the memory effect was larger for schema associations than for noschema ones ("positive interaction schema by memory").

These findings suggest that retrieval of newly learned schemarelated associations is mediated by processes in the right middle frontal, inferior frontal, and superior parietal gyrus.

\section{fMRI: connectivity}

We next examined whether interactions between regions are related to correct retrieval of the newly learned schema-related associations. Connectivity analyses were performed using two seed regions. The first seed region was centered at the activation peak within the frontal cortex, which showed increased activity in relation to retrieval of newly learned schema associations (right middle frontal gyrus: $x, y, z=24,18,52)$. Enhanced connectivity between this region and precuneus and right dorsal angular gyrus, extending into the superior parietal gyrus, was observed during correct retrieval relative to incorrect retrieval of newly learned associations on the schema board (Fig. 4). No differential connectivity was observed when comparing correct with incorrect retrieval on the noschema board.

The second seed region was based on a previous study on schema-related retrieval (van Kesteren et al., 2010b) and was centered in the $\operatorname{mPFC}(x, y, z=-6,34,12)$. This region overlaps with

the observed activity related to successful retrieval (Fig. 3A). Connectivity analyses revealed increased connectivity between the seed region and locally surrounding areas as well as the posterior cingulate cortex (PCC) and precuneus during correct retrieval relative to incorrect retrieval of newly learned associations on the schema board (Fig. 5B). When comparing correct with incorrect retrieval on the noschema board, increased connectivity was only observed with the left middle occipital gyrus (Fig. 5C). Contrasting the results of these two analyses revealed a schemarelated enhancement of mPFC-PCC/precuneus connectivity during correct compared with incorrect retrieval (Fig. 5D).

These analyses showed that enhanced connectivity between the right middle frontal gyrus, and the lateral and medial parietal cortex, as well as increased interactions between the mPFC and posterior midline structures is related to correct retrieval of newly learned, schema-related associations.

\section{Discussion}

To examine the impact of a spatial associative schema on learning and retrieval of new object-location associations in humans, we developed a paradigm conceptually linked to rodent studies. Our main findings are that: (1) the existence of a spatial associative schema made learning and retrieval of new, related associations more accurate, but slower and (2) this retrieval was associated with enhanced activation within the middle frontal, inferior frontal, and right superior parietal gyri, as well as increased connectivity between the right middle frontal gyrus, and lateral and medial posterior regions, and between the medial prefrontal cortex and posterior midline regions. Furthermore, extending previous findings (Maguire et al., 1999; van Kesteren et al., 2010a,b, 2013), retrieval of schema-defining associations is related to processes in anterior and posterior midline structures, as well as the angular gyrus.

Early psychological studies (Anderson, 1984; Ghosh and Gilboa, 2014) on schema have suggested that a schema may influence retrieval by allowing orderly memory search and facilitating reconstruction of information from memory. More orderly, or strategic, memory search may underlie retrieval of the newly learned schema-related associations, as retrieval was not only more accurate, but slower. In studies on reaction time models (Diller et al., 2001; Nobel and Shiffrin, 2001), slower cued recall than recognition was assigned to sequential search processes. Here, the schema could enable more elaborate memory search and thus slower responses. In contrast to a previous study testing schema effects on retrieval during item memory (van Kesteren et al., 2010b), the use of a more complex acquired spatial associative schema in our study is more likely to evoke such strategic retrieval processes and may explain why these effects have not previously been detected.

The frontal and parietal activity increase during schemarelated retrieval may also point toward more strategic memory search processes. Enhanced activity within the parietal lobe was mainly observed in the superior parietal gyrus, which is not only involved in visuospatial attention (Corbetta and Shulman, 2002), 
Table 2. Activity during retrieval of newly learned associations

\begin{tabular}{|c|c|c|c|c|c|}
\hline \multirow[b]{2}{*}{ Brain region } & \multicolumn{3}{|c|}{ MNI coordinates } & \multirow[b]{2}{*}{ Z-score } & \multirow[b]{2}{*}{ Voxels } \\
\hline & $x$ & $y$ & $z$ & & \\
\hline \multicolumn{6}{|l|}{ Memory:correct $>$ incorrect } \\
\hline L superior frontal gyrus & -7 & 42 & -4 & 7.18 & 1060 \\
\hline R anterior cingulate cortex & 7 & 38 & -4 & 6.81 & \\
\hline L superior frontal gyrus & 0 & 56 & 4 & 6.67 & \\
\hline L middle frontal gyrus & -60 & -63 & 10 & 6.99 & 869 \\
\hline L angular gyrus & -49 & -70 & 32 & 6.77 & \\
\hline L angular gyrus & -49 & -60 & 18 & 6.71 & \\
\hline L middle orbitofrontal gyrus & -10 & 7 & -10 & 6.91 & 235 \\
\hline L putamen & 24 & 4 & -10 & 6.21 & \\
\hline L putamen & -28 & -10 & 4 & 5.74 & \\
\hline L precuneus & -10 & -49 & 35 & 6.87 & 232 \\
\hline R posterior cingulate cortex & 7 & -49 & 32 & 6.33 & \\
\hline R middle temporal gyrus & 60 & -38 & -7 & 6.61 & 535 \\
\hline R angular gyrus & 56 & -60 & 24 & 6.59 & \\
\hline R middle temporal gyrus & 66 & -46 & -4 & 6.48 & \\
\hline Cerebellum & 21 & -49 & -24 & 6.33 & 283 \\
\hline R caudate & 10 & 10 & -7 & 6.18 & 101 \\
\hline L postcentral gyrus & -28 & -35 & 52 & 6.08 & 148 \\
\hline L precentral gyrus & -32 & -21 & 56 & 5.89 & \\
\hline Cerebellum & 24 & -52 & -52 & 5.73 & 34 \\
\hline R posterior cingulate cortex & 4 & -21 & 42 & 5.70 & 186 \\
\hline L superior frontal gyrus & -4 & -14 & 52 & 5.53 & \\
\hline R superior frontal gyrus & 7 & -7 & 52 & 4.89 & \\
\hline Cerebellum & 21 & -80 & -32 & 5.65 & 134 \\
\hline Cerebellum & 24 & -84 & -32 & 5.60 & 78 \\
\hline R precentral gyrus & 38 & -14 & 56 & 5.51 & 28 \\
\hline R precentral gyrus & 21 & -18 & 60 & 4.56 & \\
\hline$R$ cuneus & 4 & -80 & 38 & 5.21 & 78 \\
\hline L superior occipital gyrus & -7 & -84 & 28 & 5.19 & \\
\hline$R$ cuneus & 7 & -84 & 24 & 4.64 & \\
\hline L middle frontal gyrus & -35 & 18 & 46 & 5.19 & 17 \\
\hline R putamen & 28 & -4 & 10 & 5.03 & 16 \\
\hline R caudate & 14 & 18 & 18 & 5.03 & 11 \\
\hline R postcentral gyrus & 35 & -28 & 52 & 5.01 & 14 \\
\hline R superior parietal gyrus & 24 & -42 & 52 & 4.95 & \\
\hline R caudate & 21 & 4 & 24 & 4.83 & 10 \\
\hline \multicolumn{6}{|l|}{ Schema:schema $>$ noschema } \\
\hline R middle frontal gyrus & 24 & 18 & 52 & 4.74 & 79 \\
\hline R superior frontal gyrus & 24 & 32 & 49 & 3.26 & \\
\hline R inferior frontal gyrus & 49 & 28 & 21 & 4.35 & 130 \\
\hline R precentral gyrus & 56 & 10 & 32 & 3.52 & \\
\hline R angular gyrus & 46 & -70 & 32 & 4.10 & 167 \\
\hline R superior parietal gyrus & 35 & -49 & 46 & 3.98 & \\
\hline R supramarginal gyrus & 52 & -28 & 46 & 3.73 & \\
\hline \multicolumn{6}{|l|}{ Interaction schema $\times$ memory } \\
\hline R superior frontal gyrus & 4 & 32 & 42 & 4.42 & 88 \\
\hline R inferior frontal gyrus & 46 & 28 & 24 & 3.90 & 71 \\
\hline
\end{tabular}

Clusters showing significant activity during retrieval of newly learned associations (Set 2). MNI coordinates represent the location of the peak voxels. The peak voxels of each cluster with the cluster size are followed by separate ( $>8 \mathrm{~mm}$ apart) maxima (maximum of 3 ) within the cluster. First part lists areas (of 10 or more voxels) showing increased activity during correct retrieval as compared to incorrect retrieval of associations on the schema and noschema board; $p<0.05 \mathrm{FWE}$-corrected for whole brain. Note that hippocampal activity was also observed after applying small volume correction. Second part lists areas showing increased activity during retrieval (correct, incorrect) of schema-related associations as compared to associations on noschema board, and the third part shows regions with increased activity during incorrect as compared to correct retrieval in the schema relative to the noschema condition. Cluster-defining threshold of $p<0.001$ and $p<0.05$ FWE-corrected critical cluster size of 62 voxels. L, Left; $R$, right.

but also in retrieval (Wagner et al., 2005) by mediating top-down attentional control underlying strategic memory search (Cabeza et al., 2008; Ciaramelli et al., 2008; Vilberg and Rugg, 2008). According to the "attention to memory model', this region is engaged by allocating top-down attention to retrieval goals and cues and initiating memory search, as well as by monitoring and evaluating the outcome (Cabeza et al., 2008; Ciaramelli et al., 2008). When presented with a retrieval cue (i.e., an object) of a schema-related association, top-down processes might be trig- gered to search not only the memories of the newly learned, but also of neighboring, schema-defining associations, resulting in a more systematic and longer search.

Increased dorsolateral frontal activity also agrees with previous studies on monitoring of memory search and evaluation processes (Fletcher and Henson, 2001; Simons and Spiers, 2003; Mitchell and Johnson, 2009; Brod et al., 2013), potentially linked to retrieval success (Donaldson et al., 2010; Hayama et al., 2012). Here, middle frontal and superior parietal activity was associated with both correct and incorrect recall, so it indeed might be that these processes are more related to monitoring of search processes, rather than to retrieval success. In addition, eye-movements might have contributed to activity in these regions. Although this cannot be ruled out, these effects are likely limited because one would expect bilateral instead of right-sided activation.

Although the frontoparietal activity might not be related to retrieval success, we did reveal enhanced connectivity of the right middle prefrontal cortex with a set of specific posterior brain regions selectively related to correct retrieval of schema-related associations. This enhanced parietal connectivity supports the suggested role of this region in memory search and argues against merely increased perceptual attention. Moreover, increased interplay with the precuneus is in line with studies showing involvement of this region in (spatial) memory retrieval (Burgess et al., 2001; Wagner et al., 2005; Cavanna and Trimble, 2006) and memory search (Sestieri et al., 2010). Together, these findings suggest that although activity might not be related directly to retrieval success, interplay between these frontal and parietal regions appears to underlie correct retrieval of the schema-related associations.

The schema effect on memory has been linked to accelerated consolidation (Tse et al., 2007; van Kesteren et al., 2010a). In line with other studies (Frankland and Bontempi, 2005; Takashima et al., 2006; Takehara-Nishiuchi and McNaughton, 2008), this acceleration of consolidation appears associated with mPFC processing and mPFC-MTL interaction (van Kesteren et al., 2010b, 2013; Tse et al., 2011; Wang et al., 2012), yet no such connectivity changes have been observed during retrieval. We found enhanced mPFC activity associated with retrieval of schema-defining associations, suggesting that these schema-memories are differently stored and can thus be retrieved via a medial prefrontally mediated process, but no changes in mPFC-MTL connectivity. Of note, Tse et al. (2011) reported enhanced mPFC activity associated with learning of new associations when intermixed with retrieval of old associations, but not when only old associations were retrieved. This may appear at odds with our finding that mPFC activation was associated with the retrieval of schemadefining associations, but it should be considered that this occurred intermixed with the retrieval of newly learned associations that may have triggered further learning. Importantly, although enhanced $\mathrm{mPFC}$ activity was related to schema and better performance, increased mPFC activity was associated with lower performance in studies on consolidation (Takashima et al., 2006, 2007). This supports the notion that the observed mPFC effects are not merely related to difficulty, but related to consolidation processes enabled by schema. However, future studies may consider manipulating task difficulty in addition to schema.

We did not reveal differential mPFC activity between newly learned schema-related and unrelated associations, nor differential mPFC interactions with the MTL or posterior representational regions. However, enhanced $\mathrm{mPFC}$ connectivity with 


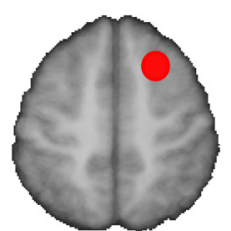

Seed: 241852

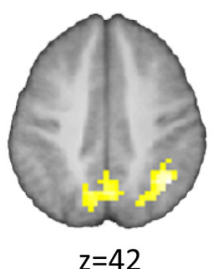

$z=42$

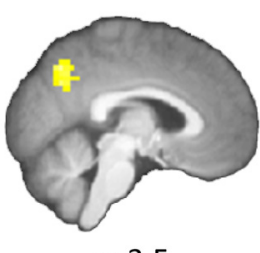

$x=3.5$

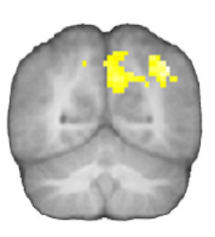

$y=-58$
5

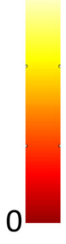

Figure 4. Connectivity changes during retrieval of schema-related associations (Set 2). Areas showing increased connectivity with the seed region in the right middle frontal gyrus, depicted as a red circle, during correct retrieval as compared with incorrect retrieval of schema-related associations. Cluster-defining threshold of $p<0.001$ and a $p<0.05$ FWE-corrected critical cluster size of 45 voxels. Connectivity changes are overlaid on a mean brain-extracted anatomical image; left $=$ left. Color bar represents $t$ values.

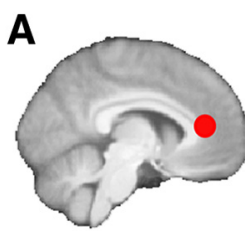

Seed: -63412

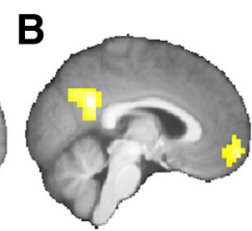

$x=3.5$

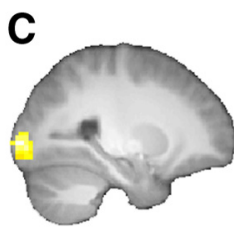

$x=24.5$

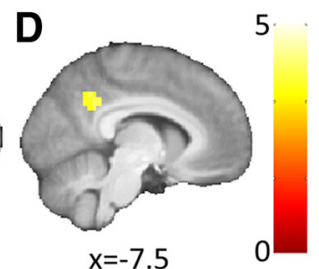

$x=-7.5$

Figure 5. Connectivity changes during retrieval of newly learned associations (Set 2). Areas showing increased connectivity with the seed region in the medial prefrontal cortex during correct as compared with incorrect retrieval. $\boldsymbol{A}$, Seed region depicted as a red circle. $\boldsymbol{B}$, Increased connectivity during retrieval of schema-related associations. $\boldsymbol{C}$, Increased connectivity during retrieval of associations on the noschema board. $\boldsymbol{D}$, Increased connectivity during retrieval of schemarelated relative to noschema associations. Cluster-defining threshold of $p<0.001$ and $p<0.05$ FWE-corrected critical cluster size of 45 voxels. Connectivity changes are overlaid on a mean brain-extracted anatomical image; left $=$ left. Color bar represents $t$ values.

posterior midline structures was related to correct retrieval of newly learned schema-related associations. This increased interaction agrees with the role of these posterior regions in general memory retrieval (Rugg and Vilberg, 2013). The absence of the expected differential mPFC activity and connectivity with representational areas might be explained by the relative novelty of our schema and the large number of to be assimilated new associations. Currently, it is still unknown how much time is needed to buildup a schema and how fast assimilation of new information occurs in humans. The augmented search and monitoring processes observed might represent an intermediate step allowing more accurate and prolonged retrieval of schema-related information before this information is fully assimilated. Furthermore, the complex nature of our task might involve many brain areas and render it more difficult to detect changes in MPFC connectivity with specific representational areas compared with studies where specific nonspatial memory is tested (van Kesteren et al., 2010b).

We found hippocampal activity associated with general correct memory retrieval, but did not find decreased hippocampal activity during retrieval of schema-defining or newly learned associations. Tse et al. (2007) showed that retrieval of newly learned schema associations rapidly became hippocampally independent. However, in humans, no decline in hippocampal activity during retrieval of schema congruent stimuli has been reported so far (van Kesteren et al., 2010b). Although we sought to develop a study more closely aligned to rodent studies, a major difference remains that rodents were not able to use the hippocampus, because of induced lesions. The hippocampus might not be necessary but, when accessible, might still contribute to retrieval of schema-related information. Moreover, our design differed from rodent studies in that the rats ran around in the arena promoting allocentric representations. Although our participants had to during the previous training session. More interference in the noschema than the schema condition is difficult to resolve, because a schema in itself may reduce interference by providing a clear structure.

Here, we aimed to align schema research in humans and rodents by using an experimentally learned spatial associative schema to investigate effects of schema on retrieval of newly learned, related associations. We propose that our results are most in line with a model in which schema improves retrieval by enabling monitoring and systematic memory search, leading to slower, but better retrieval. These augmented processes appear to be mediated by the dorsolateral prefrontal and lateral parietal cortices, as well as interactions between dorsolateral prefrontal and parietal regions, and between the medial prefrontal cortex and posterior midline structures. Our data are not conclusive about the role of the medial prefrontal cortex in schema-related retrieval. Our study-test delay might have been too short to allow full assimilation into neocortical representations requiring further consolidation processes. Thus, the augmented search and monitoring processes might represent an intermediate step allowing more accurate, but slower retrieval of schema-related information. The medial prefrontal activity observed for the retrieval of schema-defining information is in line with such an interpretation. Future studies, however, are required to examine this dynamic assimilation in more detail. Regardless of this need for future studies, our data show how retrieval of schema-related associations can be improved by additional monitoring and memory search processes. Furthermore, together with the eventarena developed for rodents, our new design might pave the way for a comprehensive translational chain from biomolecular to educational studies. 


\section{References}

Anderson JR (1981) Effects of prior knowledge on memory for new information. Mem Cogn 9:237-246. CrossRef

Anderson R (1984) Role of the reader's schema in comprehension, learning, and memory. In: Theoretical models and processes of reading, Ed 4 (Anderson R, Osborn J, Tierney R, eds). Hillsdale, NJ: Erlbaum.

Bartlett FC (1932) Remembering: a study in experimental and social psychology. Cambridge, England: UP.

Brod G, Werkle-Bergner M, Shing YL (2013) The influence of prior knowledge on memory: a developmental cognitive neuroscience perspective. Front Behav Neurosci 7:139. CrossRef Medline

Burgess N, Maguire EA, Spiers HJ, O’Keefe J (2001) A temporoparietal and prefrontal network for retrieving the spatial context of lifelike events. Neuroimage 14:439-453. CrossRef Medline

Cabeza R, Ciaramelli E, Olson IR, Moscovitch M (2008) The parietal cortex and episodic memory: an attentional account. Nat Rev Neurosci 9:613625. CrossRef Medline

Cavanna AE, Trimble MR (2006) The precuneus: a review of its functional anatomy and behavioural correlates. Brain 129:564-583. CrossRef Medline

Ciaramelli E, Grady CL, Moscovitch M (2008) Top-down and bottom-up attention to memory: a hypothesis (AtoM) on the role of the posterior parietal cortex in memory retrieval. Neuropsychologia 46:1828-1851. CrossRef Medline

Corbetta M, Shulman GL (2002) Control of goal-directed and stimulusdriven attention in the brain. Nat Rev Neurosci 3:201-215. CrossRef Medline

Diller DE, Nobel PA, Shiffrin RM (2001) An ARC-REM model for accuracy and response time in recognition and recall. J Exp Psychol Learn Mem Cogn 27:414-435. CrossRef Medline

Donaldson DI, Wheeler ME, Petersen SE (2010) Remember the source: dissociating frontal and parietal contributions to episodic memory. J Cogn Neurosci 22:377-391. CrossRef Medline

Fletcher PC, Henson RN (2001) Frontal lobes and human memory: insights from functional neuroimaging. Brain 124:849-881. CrossRef Medline

Frankland PW, Bontempi B (2005) The organization of recent and remote memories. Nat Rev Neurosci 6:119-130. CrossRef Medline

Friston KJ, Frith CD, Turner R, Frackowiak RS (1995) Characterizing evoked hemodynamics with fMRI. Neuroimage 2:157-165. CrossRef Medline

Friston KJ, Buechel C, Fink GR, Morris J, Rolls E, Dolan RJ (1997) Psychophysiological and modulatory interactions in neuroimaging. Neuroimage 6:218-229. CrossRef Medline

Ghosh VE, Gilboa A (2014) What is a memory schema? A historical perspective on current neuroscience literature. Neuropsychologia 53:104-114. CrossRef Medline

Hayama HR, Vilberg KL, Rugg MD (2012) Overlap between the neural correlates of cued recall and source memory: evidence for a generic recollection network? J Cogn Neurosci 24:1127-1137. CrossRef Medline

Kroes MC, Fernández G (2012) Dynamic neural systems enable adaptive, flexible memories. Neurosci Biobehav Rev 36:1646-1666. CrossRef Medline

Kumaran D, Summerfield JJ, Hassabis D, Maguire EA (2009) Tracking the emergence of conceptual knowledge during human decision making. Neuron 63:889-901. CrossRef Medline

Maguire EA, Frith CD, Morris RG (1999) The functional neuroanatomy of comprehension and memory: the importance of prior knowledge. Brain 122:1839-1850. CrossRef Medline

McClelland JL, McNaughton BL, O’Reilly RC (1995) Why there are complementary learning systems in the hippocampus and neocortex: insights from the successes and failures of connectionist models of learning and memory. Psychol Rev 102:419-457. CrossRef Medline

McKenzie S, Robinson NT, Herrera L, Churchill JC, Eichenbaum H (2013) Learning causes reorganization of neuronal firing patterns to represent related experiences within a hippocampal schema. J Neurosci 33:1024310256. CrossRef Medline

Mitchell KJ, Johnson MK (2009) Source monitoring 15 years later: what have we learned from fMRI about the neural mechanisms of source memory? Psychol Bull 135:638-677. CrossRef Medline
Nobel PA, Shiffrin RM (2001) Retrieval processes in recognition and cued recall. J Exp Psychol Learn Mem Cogn 27:384-413. CrossRef Medline

Poser BA, Versluis MJ, Hoogduin JM, Norris DG (2006) BOLD contrast sensitivity enhancement and artifact reduction with multiecho EPI: parallel-acquired inhomogeneity-desensitized fMRI. Magn Reson Med 55:1227-1235. CrossRef Medline

Rugg MD, Vilberg KL (2013) Brain networks underlying episodic memory retrieval. Curr Opin Neurobiol 23:255-260. CrossRef Medline

Sestieri C, Shulman GL, Corbetta M (2010) Attention to memory and the environment: functional specialization and dynamic competition in human posterior parietal cortex. J Neurosci 30:8445-8456. CrossRef Medline

Simons JS, Spiers HJ (2003) Prefrontal and medial temporal lobe interactions in long-term memory. Nat Rev Neurosci 4:637-648. CrossRef Medline

Takashima A, Petersson KM, Rutters F, Tendolkar I, Jensen O, Zwarts MJ, McNaughton BL, Fernández G (2006) Declarative memory consolidation in humans: a prospective functional magnetic resonance imaging study. Proc Natl Acad Sci U S A 103:756-761. CrossRef Medline

Takashima A, Nieuwenhuis IL, Rijpkema M, Petersson KM, Jensen O, Fernández G (2007) Memory trace stabilization leads to large-scale changes in the retrieval network: a functional MRI study on associative memory. Learn Mem 14:472-479. CrossRef Medline

Takehara-Nishiuchi K, McNaughton BL (2008) Spontaneous changes of neocortical code for associative memory during consolidation. Science 322:960-963. CrossRef Medline

Tse D, Langston RF, Kakeyama M, Bethus I, Spooner PA, Wood ER, Witter MP, Morris RG (2007) Schemas and memory consolidation. Science 316:76-82. CrossRef Medline

Tse D, Takeuchi T, Kakeyama M, Kajii Y, Okuno H, Tohyama C, Bito H, Morris RG (2011) Schema-dependent gene activation and memory encoding in neocortex. Science 333:891-895. CrossRef Medline

Tzourio-Mazoyer N, Landeau B, Papathanassiou D, Crivello F, Etard O, Delcroix N, Mazoyer B, Joliot M (2002) Automated anatomical labeling of activations in SPM using a macroscopic anatomical parcellation of the MNI MRI single-subject brain. Neuroimage 15:273-289. CrossRef Medline

van Kesteren MT, Fernández G, Norris DG, Hermans EJ (2010a) Persistent schema-dependent hippocampal-neocortical connectivity during memory encoding and postencoding rest in humans. Proc Natl Acad Sci U S A 107:7550-7555. CrossRef Medline

van Kesteren MT, Rijpkema M, Ruiter DJ, Fernández G (2010b) Retrieval of associative information congruent with prior knowledge is related to increased medial prefrontal activity and connectivity. J Neurosci 30:1588815894. CrossRef Medline

van Kesteren MT, Ruiter DJ, Fernández G, Henson RN (2012) How schema and novelty augment memory formation. Trends Neurosci 35:211-219. CrossRef Medline

van Kesteren MT, Beul SF, Takashima A, Henson RN, Ruiter DJ, Fernández G (2013) Differential roles for medial prefrontal and medial temporal cortices in schema-dependent encoding: from congruent to incongruent. Neuropsychologia 51:2352-2359. CrossRef Medline

van Kesteren MT, Rijpkema M, Ruiter DJ, Morris RG, Fernández G (2014) Building on prior knowledge: schema-dependent encoding processes relate to academic performance. J Cogn Neurosci 26:2250-2261. CrossRef Medline

Vilberg KL, Rugg MD (2008) Memory retrieval and the parietal cortex: a review of evidence from a dual-process perspective. Neuropsychologia 46:1787-1799. CrossRef Medline

Wagner AD, Shannon BJ, Kahn I, Buckner RL (2005) Parietal lobe contributions to episodic memory retrieval. Trends Cogn Sci 9:445-453. CrossRef Medline

Wang SH, Morris RG (2010) Hippocampal-neocortical interactions in memory formation, consolidation, and reconsolidation. Annu Rev Psychol 61:49-79. CrossRef Medline

Wang SH, Tse D, Morris RG (2012) Anterior cingulate cortex in schema assimilation and expression. Learn Mem 19:315-318. CrossRef Medline

Warren DE, Jones SH, Duff MC, Tranel D (2014) False recall is reduced by damage to the ventromedial prefrontal cortex: implications for understanding the neural correlates of schematic memory. J Neurosci 34:76777682. CrossRef Medline 